\section{A Scanning Probe Microscope in My Scanning Electron Microscope?}

\author{
George J. Collins, Ph.D, Huddee Ho, TopoMetrix
}

Scanning probe microscopes (SPMs) designed to fit into scanning electron microscopes (SEMs) are now becoming commercially available and you might ask, "Why would I want to put an SPM in my SEM"? The primary reason is that the two forms of microscope are very complimentary. Each microscope extends the power of the other. The SEM can do things that are hard to do with an SPM, and vice versa.

Not long after the introduction of the STM ${ }^{1}$ and the AFM ${ }^{2}$, a few researchers built custom SPMs and installed them in their SEMs. The reports of these projects to build hybrid microscopes and examples of the data they produced can be found in the scientific literature. ${ }^{3,4}$ Scanning probe microscopy is a generic term that describes a family of closely related microscopes that operate by scanning a tiny, sharp sensor probe across the surface of a specimen. By simply changing the type of probe used in the SPM or the sensing mechanism, a wide variety of physical, mechanical, electrical and chemical properties of a specimen can be measured. If these properties are recorded as the SPM records a topographic image of an area of the specimen, a high resolution map of that property will be recorded. Because the two images are recorded simultaneously, this map of that property will have pixel-topixel correspondence with the topographic image allowing correlation of the property with topographic features seen on the sample. This is analogous to acquiring an EDX map of elemental composition along with an SEM micrograph.

When a probe that can measure forces is installed in a SPM, the SPM is referred to as an atomic force microscope (AFM). Other probes or sensors can measure friction (FFM), electrical properties (EFM), magnetic forces (MFM), Thermal properties (ThM) with nanometer scale resolution. More than thirty types of probes, each capable of measuring and mapping different properties of the specimen, have been demonstrated.

\section{The SEM:}

The SEM is a powerful research tool because, among other things, it can: - quickly survey a specimen at low magnification to find a feature of interest then quickly zoom to higher magnification to study that area with submicron resolution.

- image areas as large as a few milimeters in $x$ and $y$.

- image deep and intricate structures with its great depth of field.

- determine elemental composition using $x$-ray analysis, and correlate the resulting composition map to topographic features seen in the SEM image

- quickly gather additional information using backscattered electrons, and secondary and Auger electron emissions.

In spite of all these strengths, there are some things which are not easily done by the SEM. The best lateral resolution routinely achievable by the SEM is in the range of a few nanometers, so it cannot easily resolve atomic and molecular scale features. Also, the electron beam of the SEM can damage the surface of polymers and other soft materials and can cause electrostatic charging of non-conductive samples. This can be a serious issue because most materials in our world are insulators. The electrostatic charge build-up defocuses the beam and reduces SEM image resolution. Coating the surface with a few nanometers of a conductive material can correct this problem, but the coating can obscure fine details. And finally, while the SEM can image deep and intricate structures, its z-resolution is poor. This greatly reduces the ability of the SEM to accurately measure the height of features. It also means that the SEM is limited in its ability to image fine structure on the surface of very flat samples, such as the polished silicon wafers which are used to make semiconductor chips.

\section{The SPM:}

The SPM scans a tiny stylus probe across the specimen and images the sample's micro-topography by recording the vertical motion of the stylus during the scan. Even though this mechanical scanning typically scans an area which is only a few microns square, the scanning is slow compared to the speed of an SEM. As a result, the SPM cannot easily perform long range, low magnification survey scans to find the features of interest. This is most easily done by the SEM. If a hybrid SEM/SPM has a precision translator, one can bring the SPM tip to the feature of interest which was found by the SEM. In this way, the hybrid instrument overcomes this limitation of the SPM.

The analytical power of the SPM comes from its ability to:

- easily produce high magnification $(>1,000,000 x)$ images with Angstrom scale lateral resolution on ultra-flat samples.

- clearly show sub-Angstrom structures as small as atomic vertical steps on ultra-flat samples. The vertical resolution of the SPM is only limited by its mechanical stability, its freedom from vibrations and the sensitivity of the microscope's position sensor to vertical movements of the probe.

- routinely make calibrated height and depth measurements. SPM detectors typically have vertical sensitivity better than $0.1 \bar{A}$.

- image non-conductors without having to coat them.

- image polymers with minimal damage using the non-contact scanning mode.

measure physical, mechanical, electrical, thermal and magnetic properties of specimens with sub-micron spatial resolution.

The Hybrid SEM/SPM:

The above features of the SPM complement the SEM either by extending its analytical capability or by overcoming some of its limitations. The SPM's most obvious extension of SEM capabilities is high resolution imaging. Figure 1 shows an atomic resolution STM image of graphite acquired in a hybrid SEM/SPM. The locations of individual carbon atoms in the top atomic layer of the graphite lattice can be seen as a hexagonal pattern. The vibration isolation in the hybrid system allowed this very high resolution image to be acquired in the SEM with the pumps running. The electron beam of the SEM was turned off to avoid interference with the STM measurement.

The difficulty of using the SPM to perform fast, low resolution survey scans to find specific features of interest has already been discussed. Figure 2 shows how a well engineered SEM/SPM can overcome this problem. The SEM image shows a mat of tangled fibers. The fibers are about $10 \mu$ in diameter. This sample would be almost impossible to survey with a long range SPM scan. The delicate SPM tip could tangle in the mat of fibers and break during a long range survey scan.

From the SEM image, one can see that the fibers are coated and that in a few areas, the coating is loose and has come off. The SEM can see where the coating has failed but it cannot measure the coating thickness or measure physical and mechanical properties of the sample at the point where the coating came off. The SEM image in the SEM/SPM allows the SPM translator to accurately position the SPM tip over an area where the coating is missing. Scanning the SPM tip over the edge of the coating allows the coating thickness to be measured as shown in figure 3. Figure 4 shows the high z-resolution of the SPM and its ability to measure vertical dimensions.

The SPM is also able to measure physical properties such as hardness, temperature, magnetic field strength and electrical potential. These measurements are difficult, if not impossible, for the SEM. Figure 5 shows a force/distance curve taken by the SPM. Force distance curves can be used to measure the hardness or compliance of the material under the SPM tip. Hard materials produce curves with a steep slope. Softer materials produce force/distance curves with a shallower slope. The slopes of these curves can differentiate hard and soft components of composite samples. If a force/distance curve is taken at each pixel in an AFM image, the hardness or compliance of a surface can be mapped. This allows the SPM to map the location of materials of different hardness in the way EDX maps elemental composition in the SEM.

These examples show the complimentary nature of the SEM and the SPM and give some reasons why someone might want to put an SPM in their SEM. Hybrid instruments clearly have more power than either instrument alone and can make significant advances in analytical microscopy. Now that they are commercially available, they will become much more commonplace and more examples of the power of the hybrid SEM/SPM will be shown. Soon, adding an SPM to an SEM could be much like adding another detector, but the versatility and power of the SPM will make it more like adding a battery of detectors.

1. G. Binnig, H. Rohrer, Ch. Gerber, and E. Weibel, Phys. Rev. Lett. 49, 57 (1982) 2. G. Binnig, C. F. Quate and Ch. Gerber, Phys. Rev. Lett. 56, 930 (1986) 3. G. Rosolen and M. Welland, Rev. Sci. Instrum. 63, 9 (1992)

4. Ch. Gerber, G. Binnig, H. Fuchs, O. Marti and H. Rohrer, Rev. Sci. Instrum. 57, 221 (1986) 


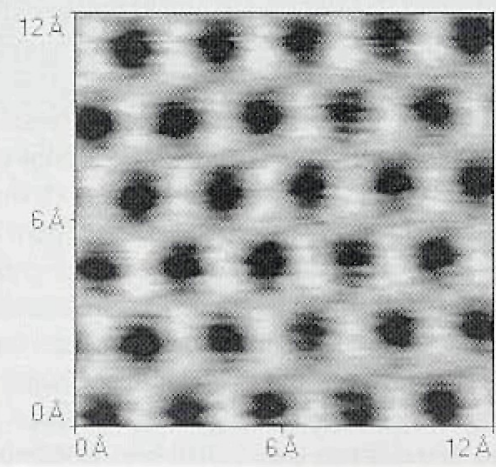

Figure 1 Atomic resolution STM Image of the surface of highly ordered pyrolytic graphite taken in a hybrid SEM/STM.

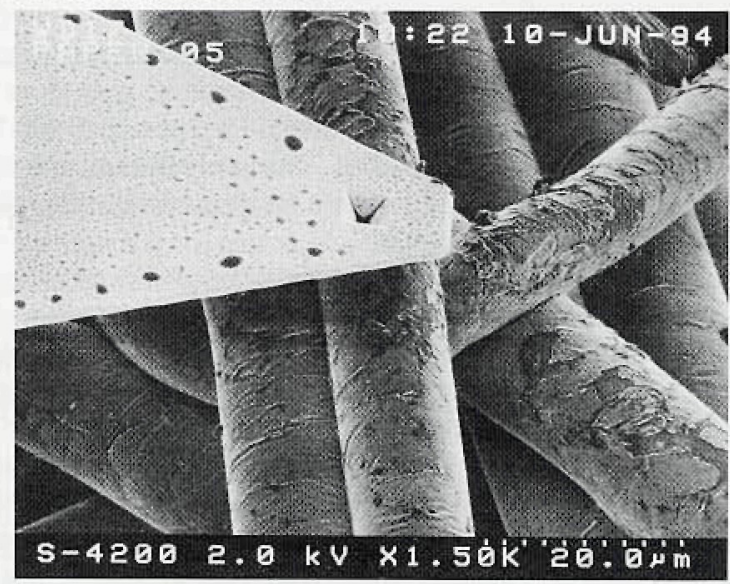

Figure 2 SEM photomicrograph showing an non-woven mat of coated 10-15 $\mu$ diameter fibers and an AFM probe. The coating appears to be poorly bound to the fibers in some areas. The pyramidal tip at the end of the AFM cantilever can be clearly seen. A precision translator moved the AFM tip to an area on a fiber where the coating had flaked off.

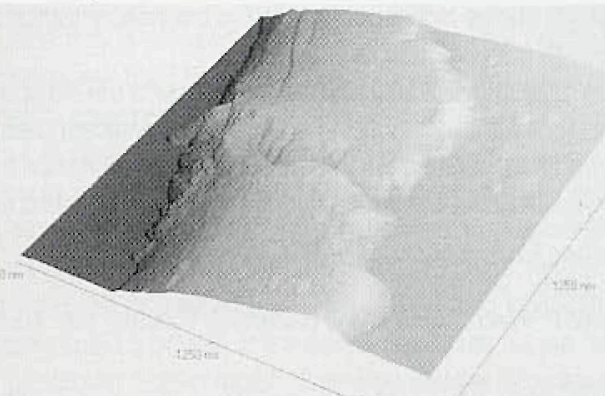

Figure 3 An AFM image of an area of a fiber where the coating had flaked off. the surface of the specimen.

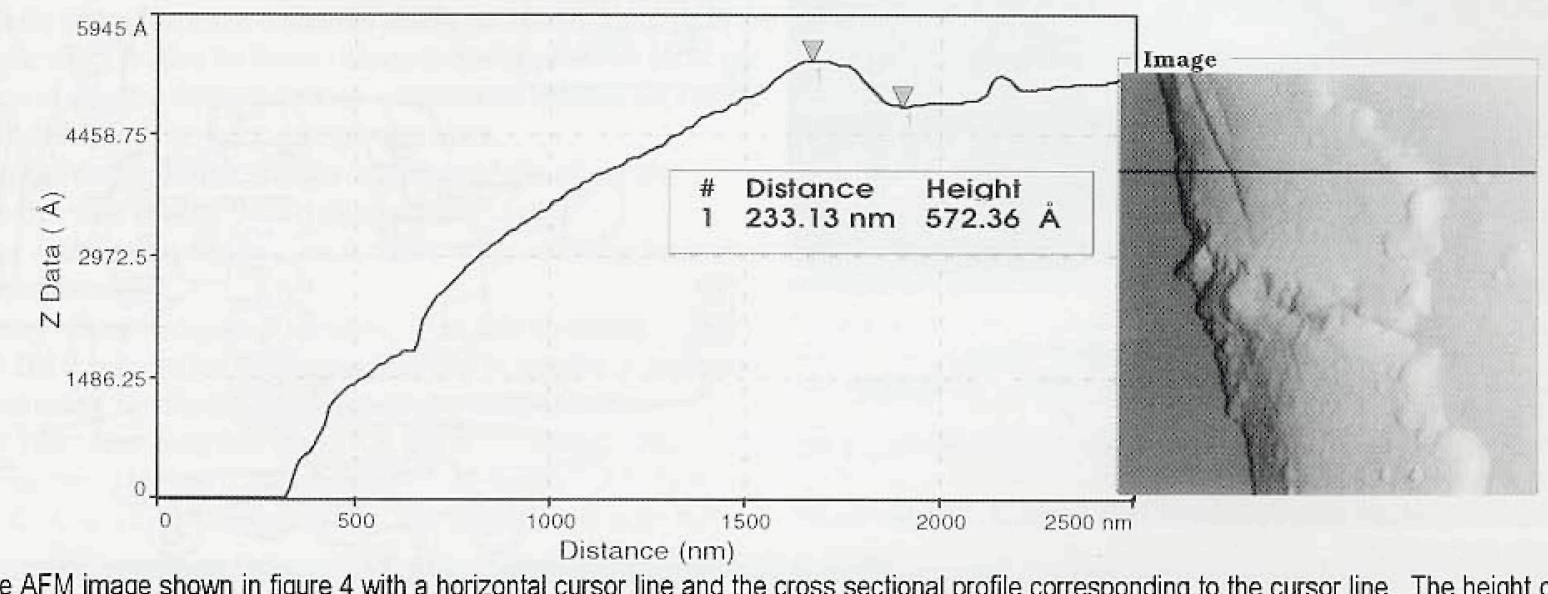

Figure 4 The AFM image shown in figure 4 with a horizontal cursor line and the cross sectional profile corresponding to the cursor line. The height of the step down from the top of the coating to the surface of the bare fiber is $572.3 \AA$.

\section{Imaging is Everything} Go digital with the SEMICAPS 4000 system-the ultimate addition to your microscopy lab!

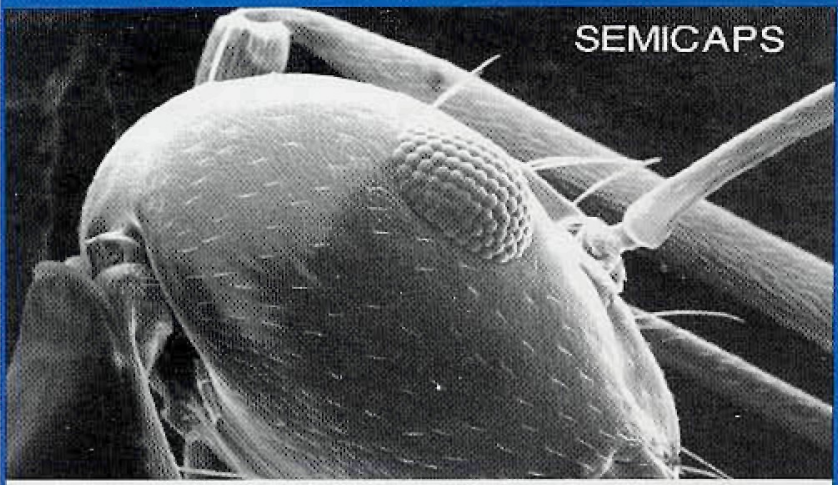

* high resolution image capture from your SEM, TEM, optical microscope, or CCD camera

* active beam, passive tap, or TV interface

* image enhancement \& processing

* $x$-ray dot mapping

* high quality, low cost printing

* image archival, search \& retrieval

* DOS \& WINDOWS applications available

$2116 \mathrm{~B}$ Walsh Avenue Santa Clara, CA 95050 Phone: (408) 986-0121 Fax: (408) 986-1059

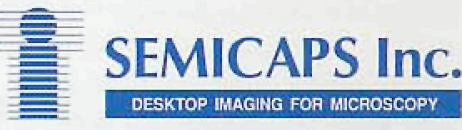

Circle Reader Response \#15

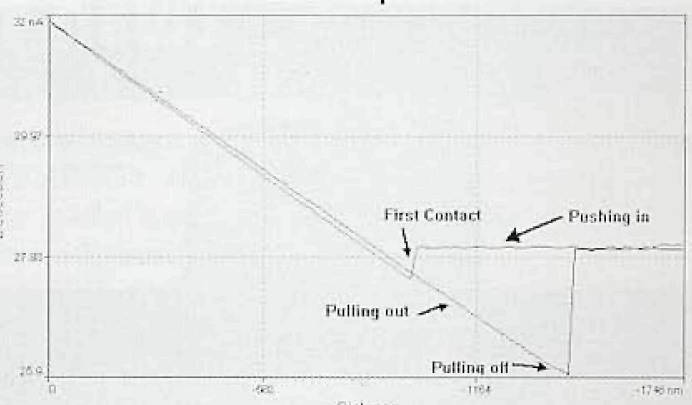

Figure 5 A force/distance curve recorded in the SEM/SPM. The slope of the diagonal line can indicate the hardness or compliance of the material directly under the tip. The downward deflection of the "pulling out" curve below the horizontal "pushing in" line can be a measure of the adhesive force between the AFM tip and 